\title{
The application of ArcGIS in error evaluation of PBN flight procedures route parameters
}

\author{
Bin Chen ${ }^{\text {a }}$, Daiwu Zhu ${ }^{\text {b }}$, Rongjun Zhang ${ }^{c}$, Yujia Liu ${ }^{\text {d }}$ \\ Civil Aviation Flight University of China, Guanghan, 618307, China \\ amartinchenbin@126.com, ${ }^{\text {bfcatcd@263.net, }{ }^{c} 449612572 @ q q . c o m,{ }^{d} 676570047 @ q q . c o m}$
}

Keywords: Performance-based Navigation (PBN), ArcGIS, Error assessment.

\begin{abstract}
In PBN flight procedure design, in order to ensure the design quality, we need to evaluation the error of route parameters. Actual test is a good way, but it often requires large amounts of human, financial and other resources. In this paper, we use ArcGIS which has powerful data analysis function to assess the route parameter error, by comparative analysis of reverser's data and design data. The results show that the error assessment methods and conclusions of the final assessment of the actual test error coincide, the method can be used to evaluation the error of route parameters in PBN flight procedure design.
\end{abstract}

\section{Introduction}

PBN (Performance Based Navigation) is a new navigation concept proposed by ICAO ${ }^{[1]}$. Introduction of PBN reflects the way from sensor-based navigation voyage to performance -based navigation changes ,the traditional ground-based navigation , aircraft navigation navigation along the ground station sends a signal through ground-based navigation aids were receiving station or back to the station flight, PBN cancel ground navigation station, geographic coordinates as waypoints, onboard navigation computer in the route parameter information stored in the flight program to calculate the current position deviation set waypoints and navigation systems ,according to given to change the mode of operation of the aircraft attitude, heading and height. So PBN for coordinate precision waypoint put forward higher requirements. In flight procedure design parameters for each waypoint coordinates are often calculated from the corresponding formula, because the earth is a spheroid, in calculating the waypoint parameters are often due to geographical differences in latitude and longitude, and the algorithm is different there will be errors . Related to the calculation of the parameters in the PBN waypoint programming, but the lack of effective error evaluation method proposed in this paper by means of ArcGIS data processing and analysis capabilities for route design parameters PBN preliminary assessment, the PBN flight procedure design route parameter error assessment has some reference value and significance.

\section{Route parameters}

Aircraft flight route called the airline, which include isometric routes and great circle route, as shown in Figure 1 and Figure 2.The great circle route is from one point on the Earth's surface to sail to another point along the great circle inferior arcs over this two voyages ${ }^{[2]}$.In PBN flight procedure design, we need to calculate the waypoint identified include: the initial approach fix, the intermediate approach fix, the final approach fix points. Mentioned here in waypoint parameters are calculated by the great circle route. PBN procedures in actual operation, the aircraft flight path depends on the definition of the actual track and roadbed coordinate navigation equipment, airborne navigation database pre-loaded flight procedures rely on navigation database coding and coordinate ${ }^{[3]}$, the implementation of point-flying, so accuracy waypoint parameters directly determine the quality of flight safety program design and aircraft flying. 


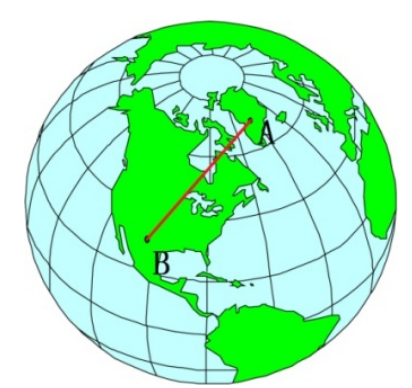

Fig.1 the great circle route

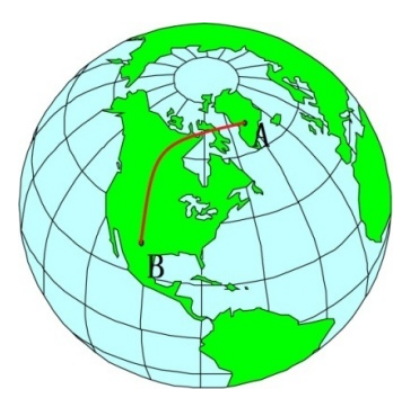

Fig.2 isometric routes

\section{ArcGIS assessment waypoint parameters}

\subsection{ArcMap}

ArcGIS is a powerful geographic information system software which developed by American Esri company, With the software, we can geographic information processing, and can be classified as secondary development ${ }^{[4]}$.ArcMap is one of the three components of ArcGIS Desktop, it is available for the data entry, editing, query, analysis, and so enabled applications, with all the features on the map, to achieve such cartography, map editing, map analysis and so on In this paper, we use ArcMap route parameters for error estimation, by using plug-in provided by ArcMap derivation and analysis data, Then we can get the error of waypoints parameters.

\subsection{Route data conversion and import}

The waypoint information in PBN design is encoded in the form of tables for publication, ArcMap supports batch import and coordinate data loading, but the latitude and longitude coordinates in degrees format, and waypoint coordinate information in the coding table are degrees minutes and seconds The format, so before you import data the format must be converted to follow a certain format in EXCEL.

Next, we use a southwest airport of approach procedures in china as an example to descripte. First collated waypoint parameters (to A1, A2, A3 ...... A10 expressed), then open ArcMap, create a new blank map, import new route data in EXCEL format.

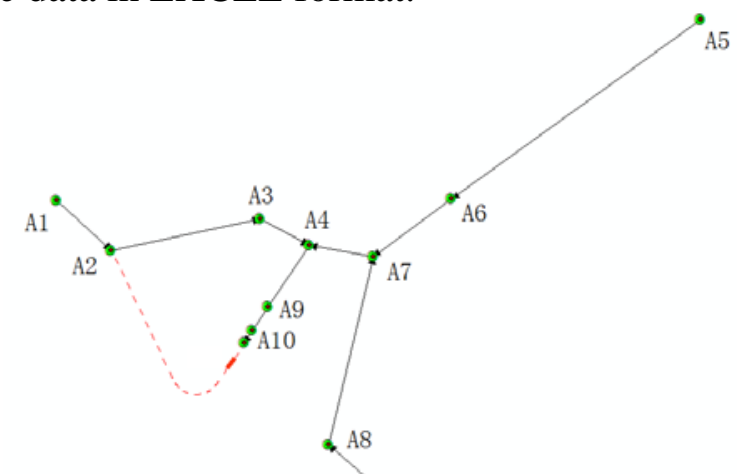

Fig. 3 waypoint coordinates load chart

\subsection{Diagram drawing program}

After importing the waypoint coordinates, then call ArcMap cartography tools, according to the demonstrated waypoint and route design information identified in the PBN program, then make each 
waypoint marking and connections. In accordance with the design standards complete chart diagram drawing program, the results shown in Figure 3.

\subsection{Route parameter estimation}

Here we use COGO tools for data analysis, COGO is an extension modules in ArcMap, which focuses on the elements of the collection After the completion of the waypoint import and dotted line connection, select the form in ArcMap editing tools in COGO which provided direction and distance data line analysis, we can get each waypoint approach procedures and the route parameters, including the direction and length information of the route, as shown in Figure 4.

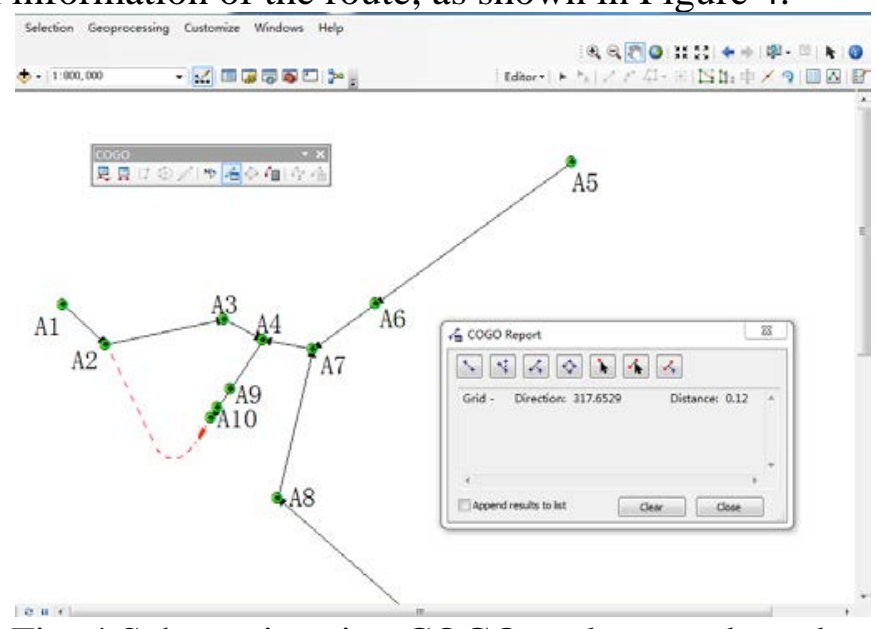

Fig. 4 Schematic using COGO tools to analyze data

Since the introduction of the waypoint coordinate information, so by analyzing the route directions and leg length information has been obtained and flight procedures have been designed for the analysis of error coding tables can be derived route errors. The direction and length of the data segment COGO conversion obtained are shown in Table 1.the PBN design data shown in Table 2. Comparison of the data in Table 1 and Table 2, we get the difference parameter table, as shown in Table 3.

Table 1 trip distance and azimuth data obtained thrust reversers

\begin{tabular}{ccc}
\hline leg & Direction/degree & length/km \\
\hline A1-A2 & 136.431 & 12.5931 \\
A2-A3 & 78.310 & 26.0252 \\
A3-A4 & 122.352 & 9.2991 \\
A5-A6 & 215.699 & 52.0967 \\
A6-A7 & 215.699 & 16.5702 \\
A7-A4 & 282.080 & 10.6161 \\
A4-A9 & 236.312 & 12.5964 \\
\hline
\end{tabular}

Table 2 PBN design trip distance and azimuth data

\begin{tabular}{ccc}
\hline leg & Direction/degree & length/km \\
\hline A1-A2 & 136.432 & 12.5930 \\
A2-A3 & 78.307 & 26.0254 \\
A3-A4 & 122.351 & 9.2990 \\
A5-A6 & 215.700 & 52.0965 \\
A6-A7 & 215.698 & 16.5703 \\
A7-A4 & 282.080 & 10.6162 \\
A4-A9 & 236.311 & 12.5961 \\
\hline
\end{tabular}

According to data obtained from the difference between the tables, we can see the direction difference of 0.001 degrees, leg length difference of about 0.1 meters, the direction difference variance is 0.000002 degree, and the length difference variance is 0.028163 meter it can be 
concluded: The current design of the route PBN point error parameters meet the requirements. If the deviation is too large, we have to reconsider the design calculation and design.

Table 3 PBN design parameters and route data ArcGIS inverse difference table

\begin{tabular}{ccc} 
leg & Direction-difference/degree & Length- difference /meter \\
A1-A2 & -0.001 & 0.1 \\
A2-A3 & 0.003 & -0.2 \\
A3-A4 & 0.001 & 0.1 \\
A5-A6 & -0.001 & 0.2 \\
A6-A7 & 0.001 & -0.1 \\
A7-A4 & 0 & -0.1 \\
A4-A9 & 0.001 & 0.3 \\
\hline
\end{tabular}

\section{Summary}

In PBN design, Route parameters calculation are commonly used, it is impossible to actually test several times to verify the route PBN flight procedures, and reliability parameters of the error, what's more, the final route will coordinate information stored parameters corresponding encoding format to guide aircraft flying in aircraft navigation database, so the error relation to the quality of route parameters and the safety of aircraft flying PBN flight procedures. In this paper, We propose to use ARCGIS comparative analysis of data, through error analysis, and the actual final PBN flight test program, Error analysis of test results and the actual results are equal, therefore the assessment method has a certain value, It can be applied to the route parameters calculation in PBN flight procedure design.

\section{Acknowledgment}

This research is supported by the Students Innovation and Entrepreneurship Training Program of Sichuan Province (Fund NO: 201410624022).

\section{References}

[1] Cai Qingyi. The technical description of performance-based navigation (PBN) [J].Air traffic management.2011 (04)

[2] Zhang Guokun. Teaching and research on isometric route, great circle route [J] map .1996, (3): 61 $\sim 64$.

[3] Zhu Daiwu, Xie Xiaofan, Liu Zhiheng. Route parameters calculation algorithm of Flight Procedures Based on Bessel geodetic [J].Science Technology and Engineering. 2013 (35).

[4] TANG Guoan, Yang Xin. ArcGIS GIS spatial analysis experiments tutorial [M]. Sciences Press, 2006. 